\title{
Sleep quality in Chinese patients with rheumatoid arthritis: contributing factors and effects on health-related quality of life
}

\section{(a) CrossMark}

Genkai Guo ${ }^{1+}$, Ting Fu ${ }^{1,2+}$, Rulan Yin ${ }^{1,2}$, Lijuan Zhang ${ }^{1,2}$, Qiuxiang Zhang ${ }^{1,2}$, Yunfei Xia', Liren Li $^{2^{*}}$ and Zhifeng $\mathrm{Gu}^{1 *}$ (D)

\begin{abstract}
Background: Poor sleep quality is common in rheumatoid arthritis (RA) patients and may lead to disease aggravation and decreased health-related quality of life (HRQoL). The increasing prevalence of poor sleep in RA patients is associated with adverse demographic, clinical, and psychological characteristics. However, there are currently no known reported studies related to the effects of sleep quality on HRQoL in RA patients from China. This cross-sectional study aims to evaluate the contributors of poor sleep and the effects of sleep quality on HRQoL in Chinese RA patients.
\end{abstract}

Methods: A self-report survey was administered to 131 RA patients and 104 healthy individuals using the Pittsburgh Sleep Quality Index (PSQI) for sleep quality. RA patients completed the Hospital Anxiety and Depression Scale for anxiety and depression, the 28-joint Disease Activity Score for disease activity, the $10 \mathrm{~cm}$ Visual Analog Scale for pain, the Health Assessment Questionnaire-Disability Index for functional capacity and the Short Form 36 health survey for HRQoL. Blood samples were taken to gain some biochemical indicators (e.g., erythrocyte sedimentation rate, C-reactive protein, rheumatoid factor, and anti-cyclic citrullinated peptide). Independent samples t-tests, Chi square analysis, logistic regression modeling and linear regression were used to analyze these data.

Results: Our results found that the prevalence of poor sleep (PSQI $\geq 5$ ) was $78.6 \%$ and the mean global score of PSQI was 7.93 (SD 3.98) in patients, which were significantly higher than the controls (18.7\% and 3.88 (SD 1.89), respectively). There were significant correlations among synthetic disease-modifying antirheumatic drugs, erythrocyte sedimentation rate, pain, disease activity, functional capacity, anxiety/depression and sleep quality in RA patients. Meanwhile, logistic regression models identified disease activity and depression as predictors of poor sleep quality. Poor RA sleepers had impaired HRQoL than good RA sleepers, and sleep quality was independently and significantly associated with social function and mental components summary.

Conclusions: The majority of Chinese RA patients suffered from poor sleep, which significantly impairs their HRQoL. The data suggested the need for holistic assessment and management of RA patients and the importance of objective interventions to improve their sleep quality and finally to improve their HRQoL.

Keywords: Rheumatoid Arthritis, Sleep quality, Quality of life, Depression, Disease activity

\footnotetext{
*Correspondence: larry017@163.com; guzhifeng@126.com

${ }^{\dagger}$ Equal contributors

${ }^{2}$ School of Nursing, Nantong University, 19th Qixiu Road, 226001 Nantong,

People's Republic of China

${ }^{1}$ Department of Rheumatology, Affiliated Hospital of Nantong University,

20th Xisi Road, 226001 Nantong, People's Republic of China
} 


\section{Background}

Rheumatoid arthritis (RA) is a chronic, inflammatory, autoimmune disorder characterized by joint pain, joint swelling and destruction of synovial joints. RA influences about $0.5-1 \%$ of adults in industrialised countries and causes disability, pain, psychological problems and decreased quality of life [1-3]. Sleep quality is an important component of health-related quality of life (HRQoL), and it has been reported that sleep problems significantly negatively affect the HRQoL in patients with RA [4]. Therefore, identifying factors contributing to poor sleep quality and studying the effects of sleep quality on HRQoL in RA patients is of great importance.

Poor sleep is one of the most prevalent and burdening symptoms and can have debilitating effect on physical and/or mental function and health [5]. It has been reported that sleep problems occur in $54 \%-70 \%$ of RA patients, including difficulty falling asleep, poor sleep quality, non restorative sleep, wakefulness, awakenings during the night and excessive daytime sleepiness $[6,7]$. Importantly, poor sleep quality may contribute to greater pain, disease activity and mood disorders, creating a cascade of dysfunction for afflicted patients [8, 9]. It has been recognized that a number of influences, such as socioeconomic status, disease activity and psychological disorders may impact sleep health, which is common in the RA population $[10,11]$.

Despite the importance of sleep quality in RA patients, only a limited number of studies related to RA patients' sleep quality have been conducted in China. The aim of this study is (1) to determine contributors of sleep quality in RA patients recruited from China; (2) to evaluate the effects of sleep quality on various dimensions of HRQoL in Chinese RA patients.

\section{Methods}

\section{Participants}

Patients who fulfilled the American College of Rheumatology (ACR) (2012) criteria for RA were recruited from the Affiliated Hospital of Nantong University from December 2014 and to December 2015 [12]. RA patients were outpatients or inpatients and healthy subjects were selected from a population attending for an annual examination. Patients were excluded based on either of the following: (1) they were less than 18 years old; (2) they did not complete questionnaires; (3) they had comorbidities (e.g., serious infections, or cardiac, respiratory, gastrointestinal, endocrine disease) that could influence disease activity; (4) they did not complete the measurements of disease activity and pain. Control subjects were excluded if they exhibited current or history of other systemic diseases or psychiatric disorders. The study was approved by the Ethics Committee of the Affiliated Hospital of Nantong
University (2014-387), and written informed consents were obtained from all of the participants, according to the Declaration of Helsinki.

\section{Demographics and clinical characteristics}

Demographic variables contain the following: body mass index (BMI), gender, age, marital status, education, occupation, and monthly income, smoking and alcohol use, which were obtained by a self-designed questionnaire. Clinical variables of disease duration, family history and medications use were obtained by asking patients or viewing medical records. Additional clinical variables of erythrocyte sedimentation rate (ESR), Creactive protein $(C R P)$, rheumatoid factor $(R F)$, and anti-cyclic citrullinated peptide (anti-CCP) were examined at the time of investigation. ESR and CRP were assessed by the Westergren method $(\mathrm{mm} / \mathrm{h})$ and the nephelometric method $(\mathrm{mg} / \mathrm{L})$, respectively. RF was measured by immunoturbidimetry using Cobas integra RFII (Roche Diagnostics GmbH, Mannheim, Germany) and anti-CCP was measured by enzyme linked immunosorbent assay (ELISA) using DIASTAT ${ }^{\mathrm{\tau} м}$ (Axis-Shield Diagnostics, Dundee, UK) [13].

Disease activity was estimated with the valid and reliable 28-joint Disease Activity Score (DAS28), incorporating 28 swollen and tender joint counts, patient's assessment of disease activity $(0-100 \mathrm{~mm}$ VAS, where $0=$ not active at all and $100=$ extremely active), ESR $(\mathrm{mm} / \mathrm{h})$ and CRP (mg/L) [14].

Pain was measured by the Visual Analogue Scale (VAS) to characterize clinical pain severity. Patients were asked to rate their experience of pain during the last week, each on a VAS of 0-10, with a higher score indicating more severe pain [15].

Functional capacity was measured by using the Health Assessment Questionnaire -Disability Index (HAQ-DI). The HAQ-DI assesses the patient's perceived difficulty in completing tasks in eight categories-dressing, arising, eating, walking, hygiene, reach, grip, and usual activities. The eight category scores were averaged into an overall HAQ-DI score and may range from 0 (no disability) to 3 (completely disabled). The Chinese HAQ-DI is a reliable and valid instrument for studies measuring disability of patients with RA [16]. "The test-retest reliability coefficient was 0.84. Between dimensions measured in HAQDI, the highest test-retest reliability was observed for walking (Spearman correlation coefficient $\mathrm{rs}=0.80$ ) and the lowest was for eating $(\mathrm{rs}=0.54)$. The internal consistency of the scale using Cronbach's alpha was high at $0.86 "$ [16].

\section{Assessment of psychological parameters}

Psychological status was measured using the Hospital Anxiety and Depression Scale (HADS), which is divided 
into HADS-A and HADS-D, both of which containing seven intermingled items [17]. Each item had a 4-point Likert scale and was scored between 0 and 3. Scores for each subscale were constructed by summation, ranging 0 - 21. The Chinese version of HADS had acceptable internal consistency and test-retest reliability, with a Cronbach alpha of 0.85 and intraclass correlation coefficient of 0.90 , respectively [18].

\section{Assessment of self-reported life quality}

Participants' health status was assessed using the Short Form 36 health survey (SF-36) in the past 4 weeks. It assessed eight domains (scores range from 0 to 100, with higher scores indicating better health status): physical function (PF); role limitations due to physical problems (RP); body pain (BP); general health perception $(\mathrm{GH})$; energy/vitality (VT); social function (SF); role limitations due to emotional problems (RE); mental health $(\mathrm{MH})$. Z-transformed and normalized domain scores were grouped into Physical Component Summary (PCS) and Mental Component Summary (MCS) [19]. The questionnaire was culturally adapted and translated into Chinese. "Convergent validity and discriminant validity were satisfactory for all except the social functioning scale. Cronbach's $\alpha$ coefficients ranged from 0.72 to 0.88 except 0.39 for the social functioning scale and 0.66 for the vitality scale. Two weeks test-retest reliability coefficients ranged from 0.66 to $0.94 "$ [20].

\section{Assessment of self-reported sleep quality}

Sleep quality was examined by the Chinese version of Pittsburgh Sleep Quality Index (PSQI) during the month preceding, which includes 19 questions completed by the subject [21]. Previous studies have found that PSQI is a well-established, commonly used measure of sleep quality that has been applied across a range of adult populations, including those with RA and other medical conditions [22]. These 19 items were broken down into the following seven components: subjective sleep quality, sleep latency, sleep duration, habitual sleep efficiency, sleep disorders, use of hypnotics, and daytime dysfunction. The seven components were each scored from 0 (no difficulty) to 3 (severe difficulty), and summed, to give an overall score ranging from 0 to 21 . Participants were dichotomized into a poor-sleep group if the PSQI was $\geq 5.0$ and a good-sleep group if the PSQI was < 5.0 [23].

\section{Data collection}

Resultant Chinese paper-based questionnaires were used to administer all instruments under physician supervision. Administration was performed in a single sitting, lasting from 15 to $20 \mathrm{~min}$. Pain and DAS28 were evaluated by the same clinician for all patients. Results were added to a computer database by two research assistants and double checked against the original data prior to analysis.

\section{Data analysis}

Descriptive statistics are provided using mean ( \pm standard deviation (SD)) or number (percentage) depending on parametric distribution of measured variables. Potential demographic, clinic and psychological variables were screened using univariate tests of the group difference (poor sleepers versus good sleepers according to the PSQI), at a lenient level of significance without correction for multiple testing (alpha $=0.05)$. The differences in terms of continuous and categorical variables that are studied in RA patients who are grouped as poor sleepers and good sleepers were evaluated with the two-tailed $t$ test and the chi-square test respectively, as well as the differences between the RA patients and the controls. All variables with a significant association with sleep quality by univariate tests were entered into a multiple stepwise logistic regression model with the dichotomous sleep quality measured by the PSQI as the dependent variable. The relations between quality of life and the other evaluation parameters were examined with Spearman rank correlation analysis. Linear regression analysis was used for each of the SF-36 components to determine independent association of sleep quality with quality of life in patients with RA after controlling for related factors, which have a significant association with each component of SF-36 by Spearman rank correlation analysis. Statistical significance was considered when $p<0.05$ (two-sided). All analyses were performed using SPSS version 20.0.

\section{Results}

\section{Patient characteristics}

As four RA patients and five healthy individuals did not complete the questionnaires, 131 RA patients (19 males and 112 females) and 104 healthy individuals (16 males and 88 females) were enrolled in the current study. Table 1 presents the baseline participant characteristics included in our study. Of the 131 RA patients included in this analysis, $85.5 \%$ were female and the mean (SD) age was 54.7(11.5) years. Almost 98.5\% RA patients were treated with synthetic Disease-Modifying Antirheumatic Drugs (DMARD) and the mean (SD) ESR was $28.47(27.64) \mathrm{mm} / \mathrm{h}$. There was no significant difference in the age, gender, BMI, marital status, education, occupation, income/month, alcohol and smoke between the RA individuals and the controls $(p>0.05)$.

\section{Self-reported sleep quality}

In the present study, we found that 103 of the 131 patients with RA had a high risk for poor sleep quality. The results demonstrated that the prevalence of poor sleep (PSQI $\geq 5$ ) 
Table 1 Baseline characteristics of RA patients and the healthy controls

\begin{tabular}{|c|c|c|c|}
\hline Variables & $\begin{array}{l}\text { Cases } \\
(n=131)\end{array}$ & $\begin{array}{l}\text { Controls } \\
(n=104)\end{array}$ & $p$ \\
\hline Gender, female, N (\%) & $112(85.5)$ & $88(84.6)$ & 0.79 \\
\hline BMI $\left(\mathrm{kg} / \mathrm{m}^{2}\right)$, Mean \pm SD & $22.5 \pm 3.12$ & $22.84 \pm 2.81$ & 0.432 \\
\hline Age (years), Mean $\pm S D$ & $54.5 \pm 11.5$ & $54.77 \pm 11.20$ & 0.851 \\
\hline Monthly income (yuan), N (\%) & & & 0.973 \\
\hline$<1000$ & $67(51.1)$ & $55(52.9)$ & \\
\hline $1000-3000$ & $47(35.9)$ & $35(33.7)$ & \\
\hline $3000-5000$ & $13(9.9)$ & $10(9.6)$ & \\
\hline$>5000$ & $4(3.1)$ & $4(3.8)$ & \\
\hline Marital status, N (\%) & & & 0.411 \\
\hline Single & $7(5.3)$ & $11(10.6)$ & \\
\hline Married & $118(90.1)$ & $90(86.5)$ & \\
\hline Divorced & $1(0.8)$ & $1(1.0)$ & \\
\hline Widowed & $5(3.8)$ & $2(1.9)$ & \\
\hline Education, N (\%) & & & 0.571 \\
\hline$\leq 9$ years & $93(71.0)$ & $70(76.9)$ & \\
\hline$>9$ years & $38(29.0)$ & $34(23.1)$ & \\
\hline Occupation, N (\%) & & & 0.757 \\
\hline Employed & $102(77.9)$ & $80(80.8)$ & \\
\hline Unemployed & $29(22.1)$ & $24(19.2)$ & \\
\hline Alcohol use, yes, N (\%) & $24(18.3)$ & $19(18.3)$ & 1.000 \\
\hline Smoking use, yes, N (\%) & $10(7.6)$ & $10(9.6)$ & 0.642 \\
\hline Disease duration (years), Mean \pm SD & $8.7 \pm 9.1$ & & \\
\hline Family history, yes, N (\%) & $10(7.6)$ & & \\
\hline \multicolumn{4}{|l|}{ Medications use, N (\%) } \\
\hline NSAIDs & $692(52.7)$ & & \\
\hline Synthetic DMARDs & $129(98.5)$ & & \\
\hline Biologic DMARDs & $11(8.4)$ & & \\
\hline Glucocorticoid & $62(47.3)$ & & \\
\hline ESR $(\mathrm{mm} / \mathrm{h})$, Mean $\pm \mathrm{SD}$ & $28.47 \pm 27.64$ & & \\
\hline CRP (mg/L), Mean \pm SD & $16.61 \pm 24.51$ & & \\
\hline RF positive, N (\%) & $102(77.9)$ & & \\
\hline anti-CCP positive, N (\%) & $91(69.5)$ & & \\
\hline DAS28, Mean \pm SD & $3.96 \pm 1.44$ & & \\
\hline HAQ-DI, Mean \pm SD & $0.50 \pm 0.61$ & & \\
\hline Total pain (VAS), Mean \pm SD & $4.32 \pm 2.76$ & & \\
\hline Nocturnal pain (VAS), Mean \pm SD & $3.95 \pm 3.22$ & & \\
\hline HADS-A, Mean \pm SD & $9.12 \pm 2.79$ & & \\
\hline HADS-D, Mean \pm SD & $8.74 \pm 2.46$ & & \\
\hline
\end{tabular}

Data are means \pm SD for continuous variables, or percentages for categorical variables

$p$ values were obtained with the chi-square test for categorical variables and the two-tailed $t$ test for continuous variables

$B M I$ Body Mass Index, NSAID Nonsteroidal anti-Inflammatory Drugs, DMARD Disease-Modifying Antirheumatic Drugs, ESR Erythrocyte Sedimentation Rate, $C R P$ C-reactive Protein, $R F$ Rheumatoid Factor, anti-CCP anti-cyclic citrullinated peptide, DAS28 Disease Activity Score in 28 Joints, HAQ-DI Health Assessment Questionnaire-Disability Index, VAS Visual Analog Scale, HADS Hospital Anxiety and Depression Scale, SF-36 the Medical Outcomes Study Short Form 36, PCS Physical Components Summary, MCS Mental Components Summary, PSQI Pittsburgh Sleep Quality Index in RA patients $(78.6 \%)$ was higher than in the controls (18.7\%) and the total PSQI score of patients with RA was significantly higher than that of the controls $(7.93 \pm 3.98$ versus $3.88 \pm 1.89 ; p<0.01$ ). Table 2 summarized the global score and the component scale scores of the PSQI for our RA sample in comparison with controls. The data showed that five of the seven components of PSQI in RA patients were significantly different from the controls: subjective sleep quality, sleep latency, habitual sleep efficiency, sleep disorders and use of hypnotics.

Differences between good and poor sleepers in RA patients As shown in Table 3, a number of demographic, clinical and psychological variables were tested for possible differences between good sleepers and poor sleepers in patients. Poor sleepers had higher disease activity scores, higher level of ESR, severer total/nocturnal pain, higher degree of depression and anxiety, with a trend toward lower functional capacity as well, compared with good sleepers $(p<0.05)$. In addition, the percentage of synthetic DMARDs use in good sleep patients was significant higher than that of poor sleep patients $(p<0.05)$.

Determinants of self-reported sleep quality in RA patients We used stepwise multiple logistic regression analysis to investigate predictors of sleep quality, as indicated in Table 4. We found that HADS-D $(\beta=0.340, p<0.01)$ and DAS28 $(\beta=0.520, p<0.01)$ were the predictors of sleep quality.

\section{Effects of self-reported sleep quality on HRQoL}

Comparison of poor sleepers and good sleepers in terms of HRQoL was summarized in Table 5. Patients with poor sleep quality had significantly lower HRQoL in all domains of the SF-36 scale. The relations between HRQoL and the other evaluation parameters were examined with Spearman rank correlation analysis (Additional file 1: Table S1). Considering observed associations of demographic, disease-related and psychological factors

Table 2 Comparison of the components of PSQI in cases and controls

\begin{tabular}{lccl}
\hline PSQI components score & Cases $(n=131)$ & Controls $(n=104)$ & $p$ \\
\hline Subjective sleep quality & $1.23 \pm 0.74$ & $0.63 \pm 0.73$ & $<0.001$ \\
Sleep latency & $1.70 \pm 1.04$ & $0.70 \pm 0.74$ & $<0.001$ \\
Sleep duration & $0.80 \pm 1.05$ & $0.70 \pm 1.00$ & 0.451 \\
Habitual sleep efficiency & $1.16 \pm 1.19$ & $0.15 \pm 0.41$ & $<0.001$ \\
Sleep disorders & $1.65 \pm 0.69$ & $0.90 \pm 0.72$ & $<0.001$ \\
Use of sleep medications & $0.52 \pm 1.04$ & $0.06 \pm 0.27$ & $<0.001$ \\
Daytime dysfunction & $0.88 \pm 0.74$ & $0.73 \pm 0.80$ & 0.148 \\
Total & $7.93 \pm 3.98$ & $3.88 \pm 1.89$ & $<0.001$ \\
\hline
\end{tabular}

Data are means (standard deviation) for continuous variables $p$ values were obtained with the two-tailed $t$ test 
Table 3 Comparison between poor and good sleepers in RA patients

\begin{tabular}{|c|c|c|c|}
\hline Variables & $\mathrm{PSQI}<5(n=28)$ & $\mathrm{PSQI} \geq 5(n=103)$ & $p$ \\
\hline Female & $22(78.6)$ & $90(87.4)$ & 0.240 \\
\hline BMI $\left(\mathrm{kg} / \mathrm{m}^{2}\right)$ & $22.88 \pm 3.40$ & $22.39 \pm 3.01$ & 0.347 \\
\hline Age (years) & $54.79 \pm 12.08$ & $54.38 \pm 11.45$ & 0.872 \\
\hline Disease duration (years) & $8.21 \pm 8.99$ & $8.83 \pm 9.15$ & 0.749 \\
\hline Monthly income (yuan) & & & 0.141 \\
\hline$<1000$ & $12(426.9)$ & $55(53.4)$ & \\
\hline $1000-3000$ & $9(32.1)$ & $38(36.9)$ & \\
\hline $3000-5000$ & $6(21.4)$ & $7(6.8)$ & \\
\hline$>5000$ & $1(3.6)$ & $3(2.9)$ & \\
\hline Marital status & & & 0.273 \\
\hline Single & $1(3.6)$ & $5(4.9)$ & \\
\hline Married & $25(89.3)$ & 94 (91.3) & \\
\hline Divorced & $1(3.6)$ & $0(0)$ & \\
\hline Widowed & $1(3.6)$ & $4(3.9)$ & \\
\hline Education & & & 0.240 \\
\hline$\leq 9$ years & $17(60.7)$ & $76(73.8)$ & \\
\hline$>9$ years & $11(39.3)$ & $27(26.2)$ & \\
\hline Occupation & & & 0.798 \\
\hline Employed & $21(75.0)$ & $81(78.6)$ & \\
\hline Unemployed & $7(25.0)$ & $22(21.4)$ & \\
\hline Smoking use, yes & $4(14.3)$ & $6(5.8)$ & 0.220 \\
\hline Alcohol use, yes & $4(14.3)$ & $20(19.4)$ & 0.783 \\
\hline Family history & $2(7.1)$ & $8(7.8)$ & 1.000 \\
\hline \multicolumn{4}{|l|}{ Medications use } \\
\hline NSAIDs & $17(60.7)$ & $52(50.5)$ & 0.396 \\
\hline Synthetic DMARDs & $26(92.9)$ & $103(100.0)$ & 0.044 \\
\hline Biologic DMARDs & $2(7.1)$ & $9(8.7)$ & 1.000 \\
\hline Glucocorticoid & $11(39.3)$ & $512(49.5)$ & 0.396 \\
\hline ESR $(\mathrm{mm} / \mathrm{h})$ & $19.14 \pm 15.96$ & $31.00 \pm 29.39$ & 0.006 \\
\hline CRP (mg/dl) & $12.36 \pm 25.00$ & $17.7668 \pm 24.37$ & 0.336 \\
\hline RF positive & $19(67.9)$ & $84(80.6)$ & 0.198 \\
\hline anti-CCP positive & $19(67.9)$ & $72(69.9)$ & 0.821 \\
\hline DAS28 & $3.15 \pm 1.39$ & $4.17 \pm 1.38$ & 0.001 \\
\hline HAQ-DI & $0.20 \pm 0.38$ & $0.52 \pm 0.65$ & 0.002 \\
\hline Total pain (VAS) & $2.96 \pm 1.86$ & $4.69 \pm 2.85$ & 0.001 \\
\hline Nocturnal pain (VAS) & $2.66 \pm 2.54$ & $4.30 \pm 3.30$ & 0.007 \\
\hline HADS-A & $8.00 \pm 2.46$ & $9.42 \pm 2.81$ & 0.016 \\
\hline HADS-D & $7.25 \pm 1.76$ & $9.03 \pm 2.49$ & 0.001 \\
\hline
\end{tabular}

Data are means $\pm S D$ for continuous variables, or percentages for categorical variables

$p$ values were obtained with the chi-square test for categorical variables and the two-tailed $t$ test for continuous variables

$B M I$ Body Mass Index, NSAID Nonsteroidal anti-Inflammatory Drugs, DMARD Disease-Modifying Antirheumatic Drugs, ESR Erythrocyte Sedimentation Rate, $C R P$ C-reactive Protein, anti-CCP anti-cyclic citrullinated peptide, $R F$ Rheumatoid Factor, DAS28 Disease Activity Score in 28 Joints, HAQ-DI Health Assessment Questionnaire-Disability Index, VAS Visual Analog Scale, HADS Hospital Anxiety and Depression Scale, SF-36 the Medical Outcomes Study Short Form 36, PCS Physical Components Summary, MCS Mental Components Summary, PSQI Pittsburgh Sleep Quality Index
Table 4 Stepwise multiple logistic regression analysis of demographic, medical and psychological variables in relation to PSQI in RA patients

\begin{tabular}{lllllll}
\hline Predictors & $\beta$ & SE & $p$ & $\operatorname{Exp}(\mathrm{B})$ & \multicolumn{2}{c}{$95 \% \mathrm{Cl}$} \\
\cline { 6 - 7 } & & & & & Lower & Upper \\
\hline HADS-D & 0.340 & 0.122 & 0.007 & 1.406 & 1.106 & 1.786 \\
DAS-28 & 0.520 & 0.193 & 0.004 & 1.681 & 1.152 & 2.453 \\
\hline
\end{tabular}

$p$ values were obtained with stepwise multiple logistic regression analysis DAS28 Disease Activity Score in 28 Joints, HADS Hospital Anxiety and Depression Scale, PSQI Pittsburgh Sleep Quality Index

with sleep quality, we conducted linear regression analysis for each of the SF-36 components to determine independent association of sleep quality with quality of life after controlling for related factors, which have a significant association with each component of SF-36 by Spearman rank correlation analysis. The results showed that sleep quality was independently and significantly associated with SF $(\beta=-0.204, p=0.012)$ and MCS $(\beta=-0.158, p=$ $0.037)$. Sleep quality was not significantly associated with the remained components of SF-36 (Table 6).

\section{Discussion}

Sleep problems are common in patients with chronic diseases and the prevalence of poor sleep is high in RA patients compared to controls [24-26]. In the current study, $78.6 \%$ of RA patients suffered from poor sleep, and disease activity and depression were independent predictors of poor sleep quality in RA patients. What is more, poor RA sleepers had impaired HRQoL than good RA sleepers and sleep quality was independently and significantly associated with HRQoL. To our knowledge, this is the first examination of the effects of sleep quality on HRQoL in Chinese RA patients.

Table 5 Comparison between poor and good sleepers in RA patients

\begin{tabular}{lccl}
\hline Variables & PSQI $<5(n=28)$ & PSQI $\geq 5(n=103)$ & $p$ \\
\hline PF & $69.82 \pm 28.07$ & $54.27 \pm 28.43$ & 0.015 \\
RP & $41.07 \pm 48.70$ & $17.23 \pm 32.09$ & 0.020 \\
BP & $60.79 \pm 24.72$ & $44.91 \pm 25.54$ & 0.004 \\
GH & $54.86 \pm 21.70$ & $38.29 \pm 19.72$ & $<0.001$ \\
VT & $63.39 \pm 17.69$ & $48.59 \pm 18.03$ & $<0.001$ \\
SF & $75.89 \pm 26.12$ & $58.62 \pm 26.55$ & 0.003 \\
RE & $54.76 \pm 46.45$ & $31.39 \pm 41.96$ & 0.012 \\
MH & $71.29 \pm 18.48$ & $58.56 \pm 17.56$ & 0.002 \\
SF-36 PCS & $56.63 \pm 24.63$ & $38.68 \pm 19.95$ & 0.001 \\
SF-36 MCS & $66.33 \pm 22.72$ & $49.30 \pm 20.27$ & 0.001 \\
\hline
\end{tabular}

$p$ values were obtained with the two-tailed $t$ test for continuous variables $P F$ physical function, $R P$ role limitations due to physical problems, $B P$ body pain, $G H$ general health perception, $V T$ energy/vitality. $S F$ social function, $R E$ role limitations due to emotional problems, $M H$ mental health, PCS Physical Components Summary, MCS Mental Components Summary, PSQI Pittsburgh Sleep Quality Index 
Table 6 Linear regression analysis of sleep quality on each of the SF-36 components

\begin{tabular}{lll}
\hline SF-36 components & $\beta$ & $p$ \\
\hline $\mathrm{PF}^{\mathrm{a}}$ & 0.057 & 0.471 \\
$\mathrm{RP}^{\mathrm{b}}$ & -0.018 & 0.836 \\
$\mathrm{BP}^{\mathrm{c}}$ & -0.060 & 0.383 \\
$\mathrm{GH}^{\mathrm{d}}$ & -0.016 & 0.067 \\
$\mathrm{VT}^{\mathrm{e}}$ & -0.097 & 0.255 \\
$\mathrm{SF}^{f}$ & -0.204 & 0.012 \\
$\mathrm{RE}^{\mathrm{g}}$ & -0.151 & 0.091 \\
$\mathrm{MH}^{\mathrm{h}}$ & -0.068 & 0.397 \\
$\mathrm{PCS}^{\mathrm{i}}$ & -0.045 & 0.527 \\
$\mathrm{MCS}^{\mathrm{g}}$ & -0.158 & 0.037 \\
\hline
\end{tabular}

$p$ values were obtained with multiple linear regression analysis a controlled for income, disease duration, ESR, CRP, DAS28, total pain, nocturnal

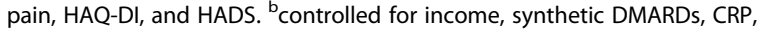
DAS28, total pain, nocturnal pain, HAQ-DI, and HADS. 'controlled for income, synthetic DMARDs, CRP, RF, DAS28, total pain, nocturnal pain, HAQ-DI, and HADS. ${ }^{d}$ controlled for age, alcohol, disease duration, synthetic DMARDs,

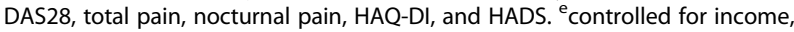
disease duration, ESR, CRP, DAS28, total pain, nocturnal pain, HAQ-DI, and HADS. ${ }^{f}$ controlled for age, income, synthetic DMARDs, ESR, CRP, RF positive, anti-CCP positive, DAS28, total pain, nocturnal pain, HAQ-DI, and HADS. ${ }^{9}$ controlled for age, income, synthetic DMARDs, ESR, CRP, RF positive, DAS28, total pain, nocturnal pain, HAQ-DI, and HADS. h'controlled for occupation, income, ESR, CRP, DAS28, total pain, nocturnal pain, HAQ-DI, and HADS. 'controlled for income, disease duration, synthetic DMARDs, ESR, CRP, DAS28, total pain, nocturnal pain, HAQ-DI, and HADS

It has been reported that several factors of demographic, clinical and psychogenic variables have an influence on sleep quality [27-29]. In our study, patients with a PSQI score $\geq 5$ were found to have significantly more frequently use synthetic DMARDs. However, Nicassio PM et al. examining sleep disturbance in RA patients have found no association between synthetic DMARDs and sleep quality [30]. While, one study has shown that YORA (age at disease onset $\leq 65$ years) patients reported more sleep disorders and received significantly more synthetic DMARDs than LORA (age at disease onset $>65$ years) RA patients [31]. In our present study, the majority of RA patients were under 65 years and $98.5 \%$ patients had synthetic DMARDs, which may account for the differences in our findings compared to other reported findings [30].

An increasing number of studies have indicated that psychological disorders are risk factors for sleep quality $[4,32,33]$. It has been reported that $20 \%-30 \%$ of RA patients have significant mood problems [9, 34]. Psychological disorders especially depression aggravate the numerous health-related comorbidities that are related to RA such as disability and limitations in quality of life $[35,36]$. In the present study, we also found that poorsleep patients had significantly higher levels of HADS-A and HADS-D scores compared with good-sleep patients. Interestingly, logistic regression analysis indicated that depression was a major contributor to sleep quality, which is consistent with previous studies [28, 30]. The data suggested the need for systemic psychiatric screening and management and the importance of targeted interventions to help RA patients get rid of sleep problems [30]. At present, some non-pharmacological treatments, such as intermittent aerobic exercise, can relieve depression and anxiety, as well as improving sleep quality in RA patients [37]. Meanwhile, it is reported that sleep management including sleep education and cognitive-behavioral interventions can improve physical/mental wellness and sleep quality [38].

Previous study has found that disease activity has a vital effect on sleep quality in RA patients [33, 34]. Westhovens et al. examining sleep problems in patients with RA have found a positive and independent association between disease activity and sleep quality [29]. Our study has shown poor-sleep patients had significantly higher levels of disease activity compared with goodsleep patients. Importantly, logistic regression analysis indicated that disease activity is a major contributor to sleep quality, which is consistent with previous studies from Turkey and Korea [4, 27]. Additionally, the ESR was found to be associated with sleep quality in our study, which was in line with prior research reports [4]. It is possible that ESR more closely correlated with polysomnography parameters recorded during sleep hypoxic episodes and moderate and severe obstructive sleep apnea were independently associated with an elevated ESR [39]. Also, there was a positive correlation between sleep quality and pain in RA patients, which was also found in our study [32]. Unexpectedly, pain did not contribute to poor sleep quality in the logistic regression analysis of our study, which was different from a previous study [30]. The possible explanation may be that the active inflammatory disease and the resulting pain are treated and the arthritic process is stopped, which resulted in less influence than other variables on sleep quality [40]. Similar to the results of Wolfe et al., the data in the present study support that functional capacity is associated with sleep quality [32]. The inability to perform the daily activities may cause depression and lead to poor sleep quality in RA patients [41]. After administration of the IL-6 receptor antagonist tocilizumab in patients with RA, changes in PSQI score over time were correlated significantly with HAQ-DI changes and marginally with changes in fatigue [42]. Therefore, IL-6 may play an important role in the mechanism of sleep quality.

HRQoL is considered as one of the main outcome measures for RA patients and sleep quality represents a central component of HRQoL in RA patients [4]. Several studies have shown that individuals with RA have poorer quality of life compared to the general population, in both the emotional and physical domains [43, 44]. We found diminished HRQoL in poor sleepers in all dimensions of 
SF-36 compared with good sleepers. Association between sleep quality remained significant for SF and MCS after controlling for demographic, disease-related and psychological factors. It is of interest that RA patients had poorer HRQoL, including general health, physical health, mental health and sleep [45]. Associations of sleep problems with pain and fatigue in RA patients are also reported by some previous studies [46]. It must be noted that fatigue and pain in RA patients are multidimensional with physical and mental health. The HRQoL instrument we used in our study was not comprehensive in this regard, which may explain our results.

Though we reported the relationship between the demographic, clinical, psychological characteristics and sleep quality and the effects of sleep quality on HRQoL in Chinese patients with RA, we also had several limitations: (1) We just measure sleep quality and psychological factors with self-report questionnaires. (2) Since the sample size was rather small and was from a single clinic of rheumatology, findings of this study cannot be generalized to all RA patients in our society. (3) The cross-sectional design of our study does not allow examining causal relationships between variables. Further studies with expanded sample sizes, objective sleep measures and prospective studies on RA patients' quality of sleep should be conducted to support the development of effective interventions to improve their sleep quality.

\section{Conclusions}

In summary, this is the first known evaluation of the contributors of poor sleep quality and the effects of sleep quality on RA patients' HRQoL in China. The present study has found that the quality of sleep was very poor in RA patients and that poor sleep quality may significantly impair their HRQoL. The results of this research provided evidence of the importance of disease activity and depression in explaining sleep quality in patients with RA. The results emphasize the need for systemic psychiatric screening, holistic assessment and targeted intervention/management of RA patients to improve their sleep quality and finally improve their HRQoL.

\section{Additional file}

Additional file 1: Table S1. Correlation coefficients between components of SF-36 and PSQI (Spearman rho). (DOC $58 \mathrm{~kb}$ )

\section{Abbreviations}

ACR: American college of rheumatology; anti-CCP: anti-Cyclic Citrullinated Peptide; BMI: Body mass index; BP: Body pain; CRP: C-reactive protein; DAS28: Disease activity score in 28 joints; DMARD: Disease-modifying antirheumatic drugs; ELISA: Enzyme linked immunosorbent assay; ESR: Erythrocyte sedimentation rate; GH: General health perception; HADS: Hospital anxiety and depression scale; HAQ-DI: Health assessment questionnaire-disability index; HRQoL: Health-related quality of life; MCS: Mental components summary; MH: Mental health; NSAID: Nonsteroidal
anti-Inflammatory Drugs; PCS: Physical components summary; PF: Physical function; PSQI: Pittsburgh sleep quality index; RA: Rheumatoid arthritis; RE: Role limitations due to emotional problems; RF: Rheumatoid factor; RP: Role limitations due to physical problems; SF: Social function; SF-36: The short form 36 health survey; VAS: Visual analog scale; VT: Energy/Vitality

\section{Acknowledgments}

We want to thank Yan Sang, Haixia Cao, Guijuan Feng, Yan Meng, Zhimin Lu, Jing Wang, Juan Ji, and Biyu Shen for their assistance with this study.

\section{Funding}

The study was supported by the College graduate research and innovation of Jiangsu Province (YKZZ15-0353) and the Nantong University Graduate Innovation Program (YKC15075)

\section{Availability of data and materials}

We have attached the databases described in the manuscript to make it available for testing by reviewers. However, we are sorry that we can't share our data now. Because we are a team and other members of our group have been doing some further researches. Some part of the results in this manuscript is the basis of their study. However, their research is not over now and their articles have not been written or published.

\section{Authors' contributions}

GG and TF have contributed to study design, data collection, data analysis, interpretation of results, preparation of the manuscript. RY, LZ, QZ and YX have contributed to data collection and data analysis; LL and ZG have contributed to study design, preparation of the manuscript. All authors read and approved the final manuscript.

\section{Competing interests}

The authors declare that they have no competing interests.

Consent for publication

Not applicable.

\section{Ethics approval and consent to participate}

The study was approved by the Ethics Committee of the Affiliated Hospital of Nantong University (2014-387), and written informed consents were obtained from all of the participants, according to the Declaration of Helsinki.

Received: 29 April 2016 Accepted: 12 October 2016

Published online: 16 November 2016

\section{References}

1. Matcham F, Scott IC, Rayner L, Hotopf M, Kingsley GH, Norton S, et al. The impact of rheumatoid arthritis on quality-of-life assessed using the SF-36: a systematic review and meta-analysis. Semin Arthritis Rheum. 2014:44:123-30.

2. Scott DL, Wolfe F, Huizinga TW. Rheumatoid arthritis. Lancet. 2010; 376(9746):1094-108.

3. Kojima M, Kojima T, Ishiguro N, Oguchi T, Oba M, Tsuchiya H, et al. Psychosocial factors, disease status, and quality of life in patients with rheumatoid arthritis. J Psychosom Res. 2009:67(5):425-31.

4. Sariyildiz MA, Batmaz I, Bozkurt M, Bez Y, Cetincakmak MG, Yazmalar L, et al. Sleep quality in rheumatoid arthritis: relationship between the disease severity, depression, functional status and the quality of life. J Clin Med Res. 2014:6(1):44-52.

5. Løppenthin K, Esbensen BA, Jennum P, Østergaard M, Tolver A, Thomsen T, et al. Sleep quality and correlates of poor sleep in patients with rheumatoid arthritis. Clin Rheumatol. 2015;34(12):2029-39.

6. Abad VC, Sarinas PS, Guilleminault C. Sleep and rheumatologic disorders. Sleep Med Rev. 2008;12(3):211-28.

7. Drewes AM, Nielsen KD, Hansen B, Taagholt SJ, Bjerregard K, Svendsen L. A longitudinal study of clinical symptoms and sleep parameters in rheumatoid arthritis. Rheumatology(Oxford). 2000;39(11):1287-9.

8. Stephenson E, Delongis A, Esdaile JM, Lehman AJ. Depressive symptoms and rheumatoid arthritis: spouse empathic responding as a buffer. Arthritis Care Res (Hoboken). 2014:66(4):532-41.

9. Covic T, Tyson G, Spencer D, Howe G. Depression in rheumatoid arthritis patients: demographic, clinical, and psychological predictors. J Psychosom Res. 2006;60(5):469-76. 
10. Xu Y, Zhang L, Huang A, Zhao Y, Liu Y. An investigation of sleep disturbance and related factors in rheumatoid arthritis patients. Zhonghua Nei Ke Za Zhi. 2015;54(11):927-30.

11. Goes AC, Reis LA, Silva MB, Kahlow BS, Skare TL. Rheumatoid arthritis and sleep quality. Rev Bras Reumatol. 2016;S0482-5004(16):30046-8.

12. Radner H, Neogi T, Smolen JS, Aletaha D. Performance of the 2010 ACR/ EULAR classification criteria for rheumatoid arthritis: a systematic literature review. Ann Rheum Dis. 2014;73(1):114-23. PMID: 23592710.

13. Kim HH, Kim J, Park SH, Kim SK, Kim OD, Choe JY. Correlation of anti-cyclic citrullinated antibody with hand joint erosion score in rheumatoid arthritis patients. Korean J Intern Med. 2010;25(2):201-6.

14. Prevoo ML, van 't Hof MA, Kuper HH, van Leeuwen MA, van de Putte LB, van Riel PL. Modified disease activity scores that include twenty-eight-joint counts. Development and validation in a prospective longitudinal study of patients with rheumatoid arthritis. Arthritis Rheum. 1995;38(1):44-8.

15. Sokka T, Kankainen A, Hannonen P. Scores for functional disability in patients with rheumatoid arthritis are correlated at higher levels with pain scores than with radiographic scores. Arthritis Rheum. 2000;43(2):386-9.

16. Koh ET, Seow A, Pong LY, Koh WH, Chan L, Howe HS, et al. Cross cultural adaptation and validation of the Chinese Health Assessment Questionnaire for use in rheumatoid arthritis. J Rheumatol. 1998;25(9):1705-8.

17. Bjelland I, Dahl AA, Haug TT, Neckelmann D. The validity of the Hospital Anxiety and Depression Scale. An updated literature review. J Psychosom Res. 2002;52(2):69-77.

18. Wang W, Chair SY, Thompson DR, Twinn SF. A psychometric evaluation of the Chinese version of the Hospital Anxiety and Depression Scale in patients with coronary heart disease. J Clin Nurs. 2009;18(13):2436-43.

19. Emery P, Kavanaugh A, Bao Y, Ganguli A, Mulani P. Comprehensive disease control (CDC): what does achieving CDC mean for patients with rheumatoid arthritis? Ann Rheum Dis. 2014;74(12):2165-74.

20. Li L, Wang HM, Shen Y. Chinese SF-36 Health Survey: translation, cultural adaptation, validation, and normalisation. J Epidemiol Community Health. 2003;57(4):259-63.

21. Liu XC, Tang MQ. Reliability and validity of the Pittsburgh sleep quality index. Chin J Psychiatry. 1996;29(02):103-7.

22. Cole JC, Motivala SJ, Buysse DJ, Oxman MN, Levin MJ, Irwin MR. Validation of a 3-factor scoring model for the Pittsburgh sleep quality index in older adults. Sleep. 2006;29(1):112-6.

23. Buysse DJ, Reynolds 3rd CF, Monk TH, Berman SR, Kupfer DJ. The Pittsburgh Sleep Quality Index: a new instrument for psychiatric practice and research. Psychiatry Res. 1989;28(2):193-213.

24. Drewes AM. Pain and sleep disturbances with special reference to fibromyalgia and rheumatoid arthritis. Rheumatology (Oxford). 1999;38(11):1035-8.

25. Sivertsen B, Krokstad S, Overland S, Mykletun A. The epidemiology of insomnia: associations with physical and mental health. The HUNT-2 study. J Psychosom Res. 2009;67(2):109-16.

26. Louie GH, Tektonidou MG, Caban-Martinez AJ, Ward MM. Sleep disturbances in adults with arthritis: prevalence, mediators, and subgroups at greatest risk. Data from the 2007 National Health Interview Survey. Arthritis Care Res (Hoboken). 2011;63(2):247-60.

27. Son CN, Choi G, Lee SY, Lee JM, Lee TH, Jeong HJ, et al. Sleep quality in rheumatoid arthritis, and its association with disease activity in a Korean population. Korean J Intern Med. 2015;30(3):384-90.

28. Purabdollah M, Lakdizaji S, Rahmani A, Hajalilu M, Ansarin K. Relationship between Sleep Disorders, Pain and Quality of Life in Patients with Rheumatoid Arthritis. J Caring Sci. 2015;4(3):233-41.

29. Westhovens R, Van der Elst K, Matthys A, Tran M, Gilloteau I. Sleep problems in patients with rheumatoid arthritis. J Rheumatol. 2014;41(1):31-40.

30. Nicassio PM, Ormseth SR, Kay M, Custodio M, Irwin MR, Olmstead R, et al. The contribution of pain and depression to self-reported sleep disturbance in patients with rheumatoid arthritis. Pain. 2012;153(1):107-12.

31. Huscher D, Sengler C, Gromnica-Ihle E, Bischoff S, Eidner T, Ochs W, et al. Clinical presentation, burden of disease and treatment in young-onset and late-onset rheumatoid arthritis: a matched-pairs analysis taking age and disease duration into account. Clin Exp Rheumatol. 2013;31(2):256-62.

32. Wolfe $\mathrm{F}$, Michaud $\mathrm{K}, \mathrm{Li}$ T. Sleep disturbance in patients with rheumatoid arthritis: evaluation by medical outcomes study and visual analog sleep scales. J Rheumatol. 2006;33(10):1942-51.

33. Nicassio PM, Ormseth SR, Custodio MK, Olmstead R, Weisman MH, Irwin MR. Confirmatory factor analysis of the Pittsburgh Sleep Quality Index in rheumatoid arthritis patients. Behav Sleep Med. 2014;12(1):1-12.
34. Moldofsky H, Lue FA, Smythe HA. Alpha EEG sleep and morning symptoms in rheumatoid arthritis. J Rheumatol. 1983;10(3):373-9.

35. Escalante A, Del Rincon I. The disablement process in rheumatoid arthritis. Arthritis Rheum. 2002:47(3):333-42.

36. Verbrugge LM, Juarez L. Profile of arthritis disability: II. Arthritis Rheum. 2006;55(1):102-13.

37. Løppenthin K, Esbensen BA, Jennum P, Østergaard M, Christensen JF, Thomsen $T$, et al. Effect of intermittent aerobic exercise on sleep quality and sleep disturbances in patients with rheumatoid arthritis- design of a randomized controlled trial. BMC Musculoskelet Disord. 2014;15(1):49-57.

38. Hideki T, Norihisa T. Sleep education with self-help treatment and sleep health promotion for mental and physical wellness in Japan. Sleep Biol Rhythms. 2016;14(1):89-99.

39. Lee WH, Wee JH, Rhee CS, Yoon IY, Kim JW. Erythrocyte sedimentation rate may help predict severity of obstructive sleep apnea. Sleep Breath. 2016;20(1):419-24.

40. Wells G, Li T, Maxwell L, Maclean R, Tugwell P. Responsiveness of patient reported outcomes including fatigue, sleep quality, activity limitation, and quality of life following treatment with abatacept for rheumatoid arthritis. Ann Rheum Dis. 2008;67(2):260-5.

41. Katz PP, Yelin EH. Activity loss and the onset of depressive symptoms: do some activities matter more than others? Arthritis Rheum. 2001:44(5):1194-202.

42. Fragiadaki K, Tektonidou MG, Konsta M, Chrousos GP, Sfikakis PP. Sleep disturbances and interleukin 6 receptor inhibition in rheumatoid arthritis. J Rheumatol. 2012;39(1):60-2.

43. Picchianti-Diamanti A, Germano V, Ferlito C, Migliore A, D'Amelio R, Laganà B. Health-related quality of life and disability in patients with rheumatoid, early rheumatoid and early psoriatic arthritis treated with etanercept. Qual Life Res. 2010;19(6):821-6

44. Hodkinson B, Musenge E, Ally M, Meyer PW, Anderson R, Tikly M. Functional disability and health-related quality of life in South Africans with early rheumatoid arthritis. Scand J Rheumatol. 2012;41(5):366-74.

45. Dominick KL, Ahern FM, Gold CH, Heller DA. Health-related quality of life among older adults with arthritis. Health Qual Life Outcomes. 2004;2:5.

46. Irwin MR, Olmstead R, Carrillo C, Sadeghi N, Fitzgerald JD, Ranganath VK, et al. Sleep loss exacerbates fatigue, depression, and pain in rheumatoid arthritis. Sleep. 2012:35(4):537-43. 